\title{
PRICING DOUBLE BARRIER PARISIAN OPTIONS USING LAPLACE TRANSFORMS
}

\author{
Céline Labart* Jérôme Lelong ${ }^{\dagger}$ \\ INRIA Paris-Rocquencourt, MathFi Project, \\ Domaine de Voluceau, Rocquencourt, \\ B.P. 105, 78153 Le Chesnay Cedex, FRANCE.
}

January 28, 2008

\begin{abstract}
In this article, we study a double barrier version of the standard Parisian options. We give closed formulas for the Laplace transforms of their prices with respect to the maturity time. We explain how to invert them numerically and prove a result on the accuracy of the numerical inversion when the function to be recovered is sufficiently smooth. Henceforth, we study the regularity of the Parisian option prices with respect to maturity time and prove that except for particular values of the barriers, the prices are of class $\mathcal{C}^{\infty}$ (see Theorem 5.1). This study heavily relies on the existence of a density for the Parisian times, so we have deeply investigated the existence and the regularity of the density for the Parisian times (see Theorem [5.4).
\end{abstract}

Keywords : double barrier option, Parisian option, Laplace transform, numerical inversion, Brownian excursions, Euler summation, option price regularity.

\section{Introduction}

The pricing and hedging of vanilla options is now part of the common knowledge and the general interest has moved on to more complex products. Practitioners need to be able to price these new products. Among them, there are the so-called path-dependent options. The ones we study in this paper are called double barrier Parisian options. They are a version with two barriers of the standard Parisian options introduced by Chesnev. Jeanblanc-Picqué. and Yor (1997). Parisian options can be seen as barrier options where the condition involves the time spent in a row above or below a certain level, and not only a hitting time. Double barrier Parisian options are options where the conditions imposed on the asset involve the time spent out of the range defined by the two barriers. In practice, Parisian options can be seen as a guarantuee against easy arbitrage: they are far less sensitive to influential agent on the market than standard barrier options. It is quite

\footnotetext{
${ }^{*}$ E-mail : labart@cmap.polytechnique.fr

${ }^{\dagger}$ E-mail : lelong@cermics.enpc.fr
} 
easy for an agent to push the price of a stock momentarily but not on a longer period so that it would affect the Parisian contract.

The valuation of single barrier Parisian options can be done by using several different methods: Monte Carlo simulations, lattices, Laplace transforms or partial differential equations. As for standard barrier options, using simulations leads to a biased problem, due to the choice of the discretisation time step in the Monte Carlo algorithm. The problem of improving the performance of Monte Carlo methods in exotic pricing has drawn much attention and has particularly been developed by Andersen and Brotherton-Ratcliffe (1996). Concerning lattices, we refer the reader to the work of Avellaneda and Wu (1999) and to Costabile (2002). An approach based on partial differential equations has been developed by Haber et al. (1999) and Wilmott (1998). We also refer to Forsvth and Vetzal (1999) for the pricing of discrete Parisian options using a PDE approach. The idea of using Laplace transforms to price single barrier Parisian options is owed to Chesnev et al. (1997). In their work, they explain how to compute these Laplace transforms. We also refer to Schröder (2003) and Hartlev (2002). The different formulas of the Laplace transforms of all the different Parisian option prices have been derived by Labart and Lelong (2005). Concerning the numerical inversion of the Laplace transforms, we refer to Abate et al. (1999) for a description of a fast and accurate numerical inversion. This algorithm is implemented by Bernard et al. (2005) and compared to a procedure for approximating a general Laplace transform with one that can be easily inverted. Moreover, there are some accuracy problems with this method as recently discussed by Abate and Valko (2004). Some other techniques for pricing Parisian options can also be found in the literature: an original concept of implied barrier was developed by Anderluh and Van der Weide (2004), the idea is to replace the Parisian option by a standard barrier option with a suitably shifted barrier.

The valuation of double barrier Parisian options has not been investigated much sofar. Baldi. Caramellino, and Iovino (2000) have proposed a method based on Monte Carlo simulations corrected by the means of sharp large deviation estimates. In this paper, we compute the prices of double barrier Parisian options by using Laplace transforms, and give some properties on the regularity of the prices. First, we give a detailed computation of the Laplace transforms of the prices with respect to the maturity time. The methodology used here significantly differs from Chesnev et al. (1997). Then, we establish a formula for the inverse of the Laplace transforms using contour integrals. Since it cannot be computed exactly, we give an upper bound of the error between the approximated price and the exact one. We improve the approximation by using the Euler summation to get a fast and accurate numerical inversion following Abate et al. (1999). The accuracy of the inversion heavily relies on the regularity of the function to be recovered. So, we naturally study the regularity of the Parisian option prices with respect to maturity time. This study in turn depends on the existence of a density for the Parisian times (see Definition 2.5), which is deeply investigated in this work. To our knowledge, the existence (or not depending on the value of the barrier) of a density for the Parisian time is a new result.

The paper is organized as follows. In Section 2] we introduce the general framework and give precise definitions of double barrier Parisian option prices (double Parisian option in short). In Section 3, we explain how to compute the price of a double Parisian option, the detailed computation is carried out in Section [4. The pricing of double Parisian options is achieved 
through numerical inversion of the Laplace transform of the prices w.r.t maturity time. As mentioned above, the technique used to prove these results is based on the regularity of the option prices and the existence of a density for the Parisian times. These two points are studied in details in Section [5. Section [6 is devoted to the inversion of the Laplace transforms and we state some results concerning the accuracy of the method.

\section{Definitions}

\subsection{Some notations}

We consider a Brownian motion $W=\left(W_{t}\right)_{t \geq 0}$ defined on a filtered probability space $(\Omega, \mathcal{F}, \mathbb{Q})$, which models a financial market. We assume that $\mathbb{Q}$ is the risk neutral measure and that $\mathcal{F}=\left(\mathcal{F}_{t}\right)_{t \geq 0}$ is the natural filtration of $W$. We denote by $T$ the maturity time. In this context, we assume that the dynamics of an asset price is given by the process $S$

$$
\forall t \in[0, T], \quad S_{t}=x \mathrm{e}^{\left(r-\delta-\sigma^{2} / 2\right) t+\sigma W_{t}},
$$

where $r>0$ is the interest rate, $\delta>0$ the dividend rate, $\sigma>0$ the volatility and $x>0$ the initial value of the stock. Working under $\mathbb{Q}$ is not that convenient, we would rather use a new probability measure $\mathbb{P}$ (introduced in Definition 2.1) which enables to integrate the drift $\left(r-\delta-\sigma^{2} / 2\right)$ into the Brownian motion itself.

Definition 2.1 (Definition of $m, \mathbb{P}$ and $Z$ ). Let $m=\frac{1}{\sigma}\left(r-\delta-\frac{\sigma^{2}}{2}\right)$ and $\mathbb{P}$ be a new probability measure, which makes $Z=\left(Z_{t}=W_{t}+m t\right)_{0 \leq t \leq T}$ a $\mathbb{P}$-Brownian motion. The change of probability is given by

$$
\left.\frac{d \mathbb{Q}}{d \mathbb{P}}\right|_{\mid \mathcal{F}_{T}}=\mathrm{e}^{m Z_{T}-\frac{m^{2}}{2} T},
$$

and under $\mathbb{P}$, the dynamics of $S$ is given by

$$
\forall t \in[0, T], \quad S_{t}=x \mathrm{e}^{\sigma Z_{t}} .
$$

Remark 2.2. Since the drift term linking $W$ and $Z$ is deterministic, $\mathcal{F}$ is also the natural filtration of $Z$.

Without any further indications, all the processes and expectations are considered under $\mathbb{P}$.

Definition 2.3 (Laplace transform). The Laplace transform of a function $f$ is defined by

$$
\hat{f}(\lambda)=\int_{0}^{+\infty} e^{-\lambda t} f(t) d t
$$

when the integral exists.

Definition 2.4 (the star notation). For any function $f$, we define the $(r, m)$ discounted value of $f$ by

$$
f^{\star}(t)=e^{\left(r+\frac{1}{2} m^{2}\right) t} f(t) .
$$

Before introducing double barrier Parisian options, we recall some definitions on excursions and on single barrier Parisian options. 


\subsection{Single barrier Parisian options}

Parisian options can be seen as barrier options where the condition involves the time spent in a row above or below a certain level and not only a hitting time. As for barrier options, which can be activated or canceled (depending on whether they are In or Out) when the asset $S$ hits the barrier, Parisian options can be activated (In options) or canceled (Out options) after $S$ has spent more than a certain time $D$ in an excursion. Such an excursion can also be described in terms of the Brownian motion $Z$. For a given barrier $L$ for the process $S$, we introduce the corresponding barrier $b$ for $Z$ defined by $b=\frac{1}{\sigma} \log \left(\frac{L}{x}\right)$.

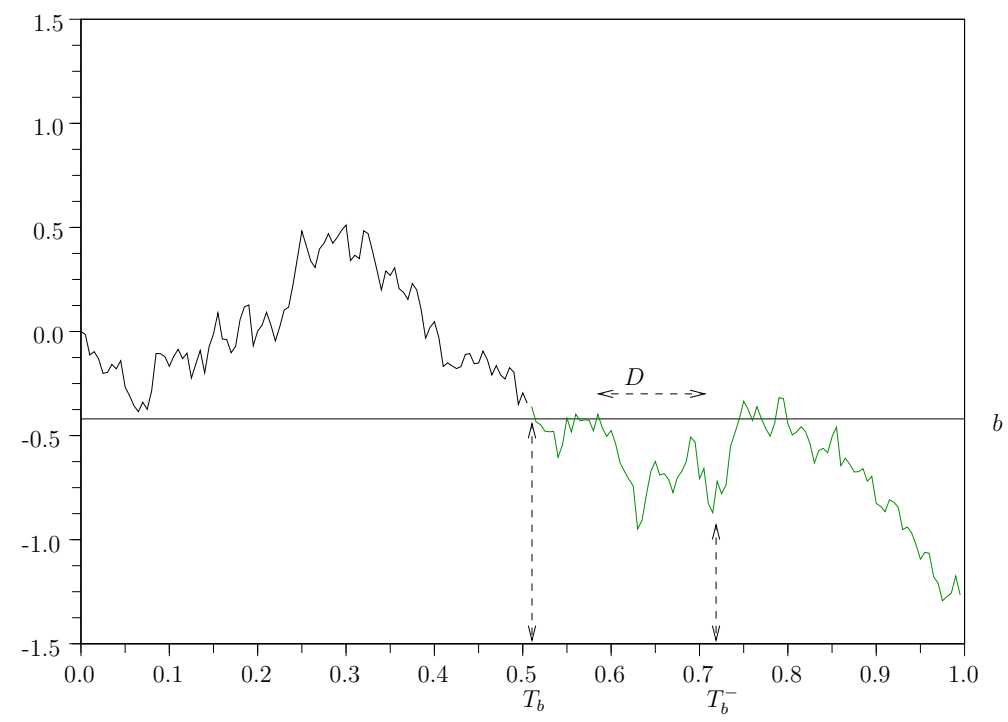

Figure 1: Brownian paths

Definition 2.5 (Parisian times $T_{b}^{-}$and $T_{b}^{+}$). For any pathwise continuous and $\mathcal{F}_{t}$-adapted process $X$, we introduce the following concepts. Let $b \in \mathbb{R}$, we define the hitting time of level $b$ by

$$
T_{b}(X)=\inf \left\{u>0: X_{u}=b\right\} .
$$

In order to define $T_{b}^{-}(X)$ (resp. $T_{b}^{+}(X)$ ) the first time the process $X$ makes an excursion longer than $D$ below (resp. above) the level $b$, we introduce $g_{t}^{b}(X)$ - the last time before $t$, $X$ has hit $b-$ for any $t>0$

$$
\begin{gathered}
g_{t}^{b}(X)=\sup \left\{u \leq t: X_{u}=b\right\} . \\
T_{b}^{-}(X)=\inf \left\{t>0:\left(t-g_{t}^{b}(X)\right) \mathbf{1}_{\left\{X_{t}<b\right\}} \geq D\right\}, \\
T_{b}^{+}(X)=\inf \left\{t>0:\left(t-g_{t}^{b}(X)\right) \mathbf{1}_{\left\{X_{t}>b\right\}} \geq D\right\} .
\end{gathered}
$$

Figure 1 represents a Brownian path which stays below $b$ longer than $D$. When no confusion is possible and the process $X$ is actually a Brownian motion, we dare write $T_{b}, T_{b}^{-}$and $T_{b}^{+}$ instead of $T_{b}(X), T_{b}^{-}(X)$ and $T_{b}^{+}(X)$. 
Definition 2.6 (Parisian Options). A Parisian option is defined by three characteristics:

- Up or Down,

- In or Out,

- Call or Put.

We use the following notations:

- PDIC stands for a Parisian Down and In call,

- PUOP stands for a Parisian Up and Out put, etc.

We recall that the price of a Parisian Down and In option with barrier $L$ is given by $e^{-r T} \mathbb{E}_{\mathbb{Q}}\left[\phi\left(S_{T}\right) \mathbf{1}_{\left\{T_{L}^{-}(S) \leq T\right\}}\right]$, where $\phi$ denotes the payoff. Using Definition 2.1, the price can be written

$$
e^{-\left(r+\frac{m^{2}}{2}\right) T} \mathbb{E}_{\mathbb{P}}\left[\mathrm{e}^{m Z_{T}} \phi\left(x \mathrm{e}^{\sigma Z_{T}}\right) \mathbf{1}_{\left\{T_{b}^{-}(Z) \leq T\right\}}\right],
$$

with $b=\frac{1}{\sigma} \log (L / x)$. Indicator $\mathbf{1}_{\left\{T_{b}^{-}(Z) \leq T\right\}}$ means that the option is activated if the Brownian motion $Z$ makes an excursion below $b$ longer than $D$ before $T$. Other single barrier Parisian options are built in the same way.

\subsection{Double barrier Parisian options}

For the sake of simplicity, we omit the word "barrier" in the following definitions of double barrier Parisian options, i.e. "double Parisian options" means "double barrier Parisian options". Double Parisian options are barrier options that are activated (or canceled) if the underlying asset $S$ stays outside a range (denoted $\left[L_{1}, L_{2}\right]$ in the following) long enough in a row.

As for single barrier Parisian options, we introduce the barriers related to the Brownian motion.

Definition 2.7 (Definition of $b_{1}, b_{2}$ and $k$ ). Let $b_{1}$ and $b_{2}$ denote the barriers corresponding to $L_{1}$ and $L_{2}$ for the Brownian motion $Z$ :

$$
b_{1}=\frac{1}{\sigma} \log \left(\frac{L_{1}}{x}\right), \quad b_{2}=\frac{1}{\sigma} \log \left(\frac{L_{2}}{x}\right) .
$$

We also introduce $k=\frac{1}{\sigma} \log \left(\frac{K}{x}\right)$.

\subsubsection{Double Parisian Out options}

A Double Parisian Out option is worthless if the asset $S$ makes an excursion outside the range $\left[L_{1}, L_{2}\right]$ older than $D$ before maturity time $T$. The price of a Double Parisian Out option at time 0 with payoff $\phi\left(S_{T}\right)$ is given by

$$
\mathrm{e}^{-r T} \mathbb{E}_{\mathbb{Q}}\left(\phi\left(S_{T}\right) \mathbf{1}_{\left\{T_{L_{1}}^{-}(S)>T\right\}} \mathbf{1}_{\left\{T_{L_{2}}^{+}(S)>T\right\}}\right) .
$$

From (2.1) and Definition 2.1] we define the price of a Double Parisian Out call. 
Definition 2.8 (Double Parisian Out call). Let $\operatorname{DPOC}\left(x, T ; K, L_{1}, L_{2} ; r, \delta\right)$ denote the value of a Double Parisian Out call. Then,

$$
D P O C\left(x, T ; K, L_{1}, L_{2} ; r, \delta\right)=\mathrm{e}^{-\left(r+\frac{1}{2} m^{2}\right) T} \mathbb{E}\left(\mathrm{e}^{m Z_{T}}\left(x \mathrm{e}^{\sigma Z_{T}}-K\right)^{+} \mathbf{1}_{\left\{T_{b_{1}}^{-}(Z)>T\right\}} \mathbf{1}_{\left\{T_{b_{2}}^{+}(Z)>T\right\}}\right) .
$$

Using the "star" notation (see Definition 2.4), we obtain

$$
\operatorname{DPOC}^{\star}\left(x, T ; K, L_{1}, L_{2} ; r, \delta\right)=\mathbb{E}\left(\mathrm{e}^{m Z_{T}}\left(x \mathrm{e}^{\sigma Z_{T}}-K\right)^{+} \mathbf{1}_{\left\{T_{b_{1}}^{-}(Z)>T\right\}} \mathbf{1}_{\left\{T_{b_{2}}^{+}(Z)>T\right\}}\right) .
$$

\subsubsection{Double Parisian In options}

The owner of a Double Parisian In option receives the payoff if $S$ makes an excursion outside the range $\left[L_{1}, L_{2}\right]$ older than $D$ before maturity time $T$. The price of a Double Parisian In option at time 0 with payoff $\phi\left(S_{T}\right)$ is given by

$$
\mathrm{e}^{-r T} \mathbb{E}_{\mathbb{Q}}\left(\phi\left(S_{T}\right) \mathbf{1}_{\left\{\left\{T_{L_{1}}^{-}(S) \leq T\right\} \cup\left\{T_{L_{2}}^{+}(S) \leq T\right\}\right\}}\right) .
$$

From (2.3), we define the price of a Double Parisian In call.

Definition 2.9 (Double Parisian In call). Let $\operatorname{DPIC}\left(x, T ; K, L_{1}, L_{2} ; r, \delta\right)$ denote the value of a Double Parisian In call. Then,

$$
D P I C\left(x, T ; K, L_{1}, L_{2} ; r, \delta\right)=\mathrm{e}^{-\left(r+\frac{1}{2} m^{2}\right) T} \mathbb{E}\left(\mathrm{e}^{m Z_{T}}\left(x \mathrm{e}^{\sigma Z_{T}}-K\right)^{+} \mathbf{1}_{\left\{\left\{T_{b_{1}}^{-}(Z) \leq T\right\} \cup\left\{T_{b_{2}}^{+}(Z) \leq T\right\}\right\}}\right) .
$$

Using the "star" notation (see Definition 2.4), we obtain

$$
D P I C^{\star}\left(x, T ; K, L_{1}, L_{2} ; r, \delta\right)=\mathbb{E}\left(\mathrm{e}^{m Z_{T}}\left(x \mathrm{e}^{\sigma Z_{T}}-K\right)^{+} \mathbf{1}_{\left\{\left\{T_{b_{1}}^{-}(Z) \leq T\right\} \cup\left\{T_{b_{2}}^{+}(Z) \leq T\right\}\right\}}\right) .
$$

\section{Valuation of Double Parisian options}

This section is devoted to the valuation of Double Parisian options. We establish several relations between the different Double Parisian option prices so that computing $D P O C^{\star}$ and $D P I C^{\star}$ comes down to evaluating $\mathbb{E}\left(\mathrm{e}^{m Z_{T}}\left(x \mathrm{e}^{\sigma Z_{T}}-K\right)^{+} \mathbf{1}_{\left\{T_{b_{1}} \leq T\right\}} \mathbf{1}_{\left\{T_{b_{2}}^{+} \leq T\right\}}\right)$. As for single barrier Parisian options, parity relationships between call and put options hold.

\subsection{Valuation of Double Parisian Out and In calls}

Before giving new formulas for $D P O C^{\star}$ and $D P I C^{\star}$, we define the "star" price of a standard call option.

Definition 3.1. Let $S C$ denote the price of a standard call option.

$$
S C^{\star}(x, T ; K ; r, \delta)=\mathbb{E}\left(\left(x \mathrm{e}^{\sigma Z_{T}}-K\right)^{+} \mathrm{e}^{m Z_{T}}\right) .
$$

Proposition 3.2. Using the notations of Section 2.2, the following relations hold

$$
\begin{aligned}
D P O C^{\star}\left(x, T ; K, L_{1}, L_{2} ; r, \delta\right)= & S C^{\star}(x, T ; K ; r, \delta)-P D I C^{\star}\left(x, T ; K, L_{1} ; r, \delta\right) \\
& -P U I C^{\star}\left(x, T ; K, L_{2} ; r, \delta\right)+A\left(x, T ; K, L_{1}, L_{2} ; r, \delta\right), \\
D P I C^{\star}\left(x, T ; K, L_{1}, L_{2} ; r, \delta\right)= & P D I C^{\star}\left(x, T ; K, L_{1} ; r, \delta\right) \\
& +P U I C^{\star}\left(x, T ; K, L_{2} ; r, \delta\right)-A\left(x, T ; K, L_{1}, L_{2} ; r, \delta\right),
\end{aligned}
$$


where

$$
\begin{array}{r}
A\left(x, T ; K, L_{1}, L_{2} ; r, \delta\right)=\mathbb{E}\left[\mathbf{1}_{\left\{T_{b_{1}}^{-} \leq T\right\}} \mathbf{1}_{\left\{T_{b_{1}}^{-} \leq T_{b_{2}}^{+}\right\}} e^{m Z_{T_{b_{1}}}} P U I C^{\star}\left(x e^{\sigma Z_{T_{b_{1}}}^{-}}, T-T_{b_{1}}^{-} ; K, L_{2} ; r, \delta\right)\right] \\
+\mathbb{E}\left[\mathbf{1}_{\left\{T_{b_{2}}^{+} \leq T\right\}} \mathbf{1}_{\left\{T_{b_{2}}^{+}<T_{b_{1}}^{-}\right\}} e^{m Z_{T_{b_{2}}^{+}} P D I C^{\star}}\left(x e^{\sigma Z_{T_{b_{2}}^{+}}^{+}}, T-T_{b_{2}}^{+} ; K, L_{1} ; r, \delta\right)\right] . \quad(3.3)
\end{array}
$$

Proof. Step 1 : Equation (3.1) ensues from Definition 2.8 and from the following equality

$$
\mathbf{1}_{\left\{T_{b_{1}}^{-}>T\right\}} \mathbf{1}_{\left\{T_{b_{2}}^{+}>T\right\}}=1-\mathbf{1}_{\left\{T_{b_{1}}^{-} \leq T\right\}}-\mathbf{1}_{\left\{T_{b_{2}}^{+} \leq T\right\}}+\mathbf{1}_{\left\{T_{b_{1}}^{-} \leq T\right\}} \mathbf{1}_{\left\{T_{b_{2}}^{+} \leq T\right\}},
$$

with

$$
A\left(x, T ; K, L_{1}, L_{2} ; r, \delta\right)=\mathbb{E}\left[e^{m Z_{T}}\left(x \mathrm{e}^{\sigma Z_{T}}-K\right)_{+} \mathbf{1}_{\left\{T_{b_{1}}^{-} \leq T\right\}} \mathbf{1}_{\left\{T_{b_{2}}^{+} \leq T\right\}}\right] .
$$

Equation (3.2) ensues from Definition[2.9] and from $\mathbf{1}_{\left\{\left\{T_{b_{1}}^{-} \leq T\right\} \cup\left\{T_{b_{2}}^{+} \leq T\right\}\right\}}=\mathbf{1}_{\left\{T_{b_{1}}^{-} \leq T\right\}}+\mathbf{1}_{\left\{T_{b_{2}}^{+} \leq T\right\}}$ $\mathbf{1}_{\left\{T_{b_{1}}^{-} \leq T\right\}} \mathbf{1}_{\left\{T_{b_{2}}^{+} \leq T\right\}}$.

Step 2 : We split Equation (3.4) into two terms depending on the relative position of $T_{b_{1}}^{-}$ and $T_{b_{2}}^{+}$. This leads to

$$
\begin{aligned}
A\left(x, T ; K, L_{1}, L_{2} ; r, \delta\right) & =\mathbb{E}\left[\mathbf { 1 } _ { \{ T _ { b _ { 1 } } ^ { - } \leq T \} } \mathbf { 1 } _ { \{ T _ { b _ { 1 } } ^ { - } < T _ { b _ { 2 } } ^ { + } \} } \mathbb { E } \left[e^{\left.\left.m Z_{T}\left(x \mathrm{e}^{\sigma Z_{T}}-K\right)_{+} \mathbf{1}_{\left\{T_{b_{2}}^{+} \leq T\right\}} \mid \mathcal{F}_{T_{b_{1}}^{-}}\right]\right]}\right.\right. \\
& +\mathbb{E}\left[\mathbf { 1 } _ { \{ T _ { b _ { 2 } } ^ { + } \leq T \} } \mathbf { 1 } _ { \{ T _ { b _ { 2 } } ^ { + } < T _ { b _ { 1 } } ^ { - } \} } \mathbb { E } \left[e^{\left.\left.m Z_{T}\left(x \mathrm{e}^{\sigma Z_{T}}-K\right)_{+} \mathbf{1}_{\left\{T_{b_{1}} \leq T\right\}} \mid \mathcal{F}_{T_{b_{2}}^{+}}\right]\right]}\right.\right. \\
& \triangleq A_{1}+A_{2} .
\end{aligned}
$$

$A_{1}$ and $A_{2}$ being almost symmetric in $b_{1}$ and $b_{2}$, we only focus on the computation of $A_{1}$. By conditioning w.r.t to $\mathcal{F}_{T_{b_{1}}^{-}}$and using the strong Markov property, we find

$A_{1}=\mathbb{E}\left[\mathbf{1}_{\left\{T_{b_{1}}^{-} \leq T\right\}} \mathbf{1}_{\left\{T_{b_{1}}^{-}<T_{b_{2}}^{+}\right.} e^{m Z_{T_{b_{1}}}} \mathbb{E}\left[\left.e^{m B_{T-\tau}}\left(x e^{\sigma\left(B_{T-\tau}+z\right)}-K\right)^{+} \mathbf{1}_{\left\{T_{b_{2}-z}^{+} \leq T-\tau\right\}}\right|_{\tau=T_{b_{1}}^{-}, z=Z_{T_{b_{1}}^{-}}}\right]\right.$,

where $B$ is a Brownian motion independent of $\mathcal{F}_{T_{b_{1}}}$. From this equality we easily deduce the first part of the r.h.s. of (3.3). A similar proof shows that $A_{2}$ is equal to the second part of the r.h.s. of (3.3).

Remark 3.3. Dealing with inequalities of the type $\mathbf{1}_{\left\{T_{b}^{ \pm} \leq T\right\}}$ is much simpler than $\mathbf{1}_{\left\{T_{b}^{ \pm}>T\right\}}$ since we can condition w.r.t. $\mathcal{F}_{T_{b}^{ \pm}}$and use the strong Markov property. That's why we have split Equation (2.2) into four terms using the prices of single barrier Parisian options.

Remark 3.4. The computation of $D P O C^{\star}$ will be done using the numerical inversion of its Laplace transform with respect to $T$. Explicit formulas for the Laplace transforms of the first three terms in (3.1) - $\widehat{S C^{\star}}, \widehat{P D I C^{\star}}, \widehat{P U I C^{\star}}$ - are recalled in Appendix D.

It remains to compute the Laplace transform of $A$ w.r.t. maturity time. 


\subsection{A Call Put parity relationship}

Proposition 3.5. The following relationships hold

$$
\begin{aligned}
\operatorname{DPOP}\left(x, T ; K, L_{1}, L_{2} ; r, \delta\right) & =x K \operatorname{DPOC}\left(\frac{1}{x}, T ; \frac{1}{K}, \frac{1}{L_{2}}, \frac{1}{L_{1}} ; \delta, r\right), \\
\operatorname{DPIP}\left(x, T ; K, L_{1}, L_{2} ; r, \delta\right) & =x K \operatorname{DPIC}\left(\frac{1}{x}, T ; \frac{1}{K}, \frac{1}{L_{2}}, \frac{1}{L_{1}} ; \delta, r\right) .
\end{aligned}
$$

The proof being the same as for single barrier Parisian options, we refer to Chesnev et al. (1997, Section 6) for more details.

\section{Computation of Laplace transforms}

The computation of $D P O C^{\star}$ and $D P I C^{\star}$ will be done using the numerical inversion of their Laplace transforms w.r.t. the maturity time. As explained in Remark 3.4 the computation of the Laplace transforms of $D P O C^{\star}$ and $D P I C^{\star}$ boils down to the one of $A$. We would like to point out that the methodology used here to compute the Laplace transforms significantly differs from the one of the pioneer work of Chesnev et al. (1997) on single Parisian options.

Theorem 4.1 (Laplace transform of Double barrier Parisian options). The following relations hold

$$
\begin{aligned}
\widehat{D P O C}^{\star}\left(x, \lambda ; K, L_{1}, L_{2} ; r, \delta\right)= & \widehat{S C}^{\star}(x, \lambda ; K ; r, \delta)-\widehat{P D I C}^{\star}\left(x, \lambda ; K, L_{1} ; r, \delta\right) \\
& -\widehat{P U I C}^{\star}\left(x, \lambda ; K, L_{2} ; r, \delta\right)+\widehat{A}\left(x, \lambda ; K, L_{1}, L_{2} ; r, \delta\right), \\
\widehat{D P I C}^{\star}\left(x, \lambda ; K, L_{1}, L_{2} ; r, \delta\right)= & \widehat{P D I C}^{\star}\left(x, \lambda ; K, L_{1} ; r, \delta\right) \\
& +\widehat{P U I C}^{\star}\left(x, \lambda ; K, L_{2} ; r, \delta\right)-\widehat{A}\left(x, \lambda ; K, L_{1}, L_{2} ; r, \delta\right),
\end{aligned}
$$

where $\widehat{A}$ is the Laplace transform of $A$ w.r.t. maturity time given by

$$
\begin{aligned}
\widehat{A}\left(x, \lambda ; K, L_{1}, L_{2} ; r, \delta\right)= & \mathbb{E}\left[e^{-\lambda T_{b_{1}}^{-}} \mathbf{1}_{\left\{T_{b_{1}}^{-}<T_{b_{2}}^{+}\right\}}\right] \mathbb{E}\left[e^{\sqrt{2 \lambda} Z_{T_{b_{1}}}}\right] \widehat{P U I C}_{\left.\right|_{x<L_{2}}}^{\star}\left(x, \lambda ; K, L_{2} ; r, \delta\right) \\
& +\mathbb{E}\left[e^{-\lambda T_{b_{2}}^{+}} \mathbf{1}_{\left\{T_{b_{2}}^{+}<T_{b_{1}}^{-}\right\}}\right] \mathbb{E}\left[e^{-\sqrt{2 \lambda} Z_{T_{b_{2}}^{+}}}\right] \widehat{P D I C} \widehat{\mid}_{x>L_{1}}^{\star}\left(x, \lambda ; K, L_{1} ; r, \delta\right),
\end{aligned}
$$

where $\widehat{P U I C}_{\left.\right|_{x<L_{2}}}^{\star}$ (resp. $\widehat{P D I C}_{\left.\right|_{x>L_{1}}}^{\star}$ ) means that we use the definition of $\widehat{P U I C}^{\star}$ (resp. $\left.\widehat{P D I C}^{\star}\right)$ in the case $x<L_{2}\left(\right.$ resp. $\left.x>L_{1}\right)$.

We refer to Appendix $\mathbb{C}$ for explicit formulas of $\mathbb{E}\left[e^{-\lambda T_{b_{1}}^{-}} \mathbf{1}_{\left\{T_{b_{1}}^{-}<T_{b_{2}}^{+}\right\}}\right], \mathbb{E}\left[e^{-\lambda T_{b_{2}}^{+}} \mathbf{1}_{\left\{T_{b_{2}}^{+}<T_{b_{1}}^{-}\right\}}\right]$, $\mathbb{E}\left[\exp \left(\sqrt{2 \lambda} Z_{T_{b_{1}}^{-}}\right)\right]$and $\mathbb{E}\left[\exp \left(-\sqrt{2 \lambda} Z_{T_{b_{2}}^{+}}\right)\right]$.

Proof. The first part of the theorem directly ensues from Proposition 3.2

Using the decomposition of $A$ given by Proposition 3.2 we can split $A$ into $A_{1}+A_{2}$. We concentrate on $\hat{A}_{1}$ as $A_{2}$ can be treated the same way. We aim at proving that

$$
\widehat{A_{1}}=\left.\mathbb{E}\left[e^{-\lambda T_{b_{1}}^{-}} \mathbf{1}_{\left\{T_{b_{1}}^{-}<T_{b_{2}}^{+}\right\}}\right] \mathbb{E}\left[\mathrm{e}^{\sqrt{2 \lambda} Z_{T_{b_{1}}^{-}}}\right] \widehat{P U I C}\right|_{\left.\right|_{x<L_{2}}} ^{\star}\left(x, \lambda ; K, L_{2} ; r, \delta\right) .
$$


Equality (4.1) ensues from the two following relations - proved hereafter -

$$
\begin{gathered}
\widehat{A_{1}}=\mathbb{E}\left[e^{-\lambda T_{b_{1}}^{-}} \mathbf{1}_{\left\{T_{b_{1}}^{-}<T_{b_{2}}^{+}\right\}}\right] \mathbb{E}\left[\mathrm{e}^{m Z_{T_{b_{1}}}} \widehat{P U I C}^{\star}\left(x \mathrm{e}^{\sigma Z_{T_{b_{1}}}^{-}}, \lambda ; K, L_{2} ; r, \delta\right)\right], \\
\mathbb{E}\left[\mathrm{e}^{m Z_{T_{b_{1}}}} \widehat{P U I C}^{\star}\left(x \mathrm{e}^{\sigma Z_{T_{b_{1}}}^{-}}, \lambda ; K, L_{2} ; r, \delta\right)\right]=\mathbb{E}\left[\mathrm{e}^{\sqrt{2 \lambda} Z_{T_{b_{1}}}}\right] \widehat{P U I C}_{\left.\right|_{x<L_{2}}}^{\star}\left(x, \lambda ; K, L_{2} ; r, \delta\right) .
\end{gathered}
$$

Step 1: Proof of (4.2).

As a function of $T, A_{1}$ shows up as a convolution, hence its Laplace transform is given by

$$
\widehat{A_{1}}=\mathbb{E}\left[e^{-\lambda T_{b_{1}}^{-}} \mathbf{1}_{\left\{T_{b_{1}}^{-}<T_{b_{2}}^{+}\right\}} e^{m Z_{T_{b_{1}}^{-}}} \widehat{P U I C}^{\star}\left(x e^{\sigma Z_{T_{b_{1}}}}, \lambda ; K, L_{2} ; r, \delta\right)\right] .
$$

From Chesnev et al. 1997, Sections 8.3 and 8.4), we know that $T_{b_{1}}^{-}$is an $\mathcal{F}_{g_{t}}^{+}$-stopping time whereas $Z_{T_{b_{1}}^{-}}$is independent of $\mathcal{F}_{g_{T_{b_{1}}^{-}}^{+}}^{+}$. Hence, Step 1 is completed.

\section{Step 2: Proof of (4.3).}

Since $x \exp \left(\sigma Z_{T_{b_{1}}^{-}}\right)<L_{2}\left(\right.$ as $\left.Z_{T_{b_{1}}^{-}}<b_{1}\right)$, we compute $\widehat{P U I C}^{\star}\left(x \exp \left(\sigma Z_{T_{b_{1}}}\right), \lambda ; K, L_{2} ; r, \delta\right)$ using the definition of $\widehat{P U I C}^{\star}\left(x, \lambda ; K, L_{2} ; r, \delta\right)$ in the case $x<L_{2}$. In such a case, we can write $\widehat{P U I C}^{\star}\left(x, \lambda ; K, L_{2} ; r, \delta\right)=x^{\frac{\sqrt{2 \lambda}-m}{\sigma}} f\left(\lambda ; K, L_{2} ; r, \delta\right)$, where $f$ can easily be deduced from the formulas recalled in Appendix D. Then, we find

$$
\mathbb{E}\left[e^{m Z_{T_{b_{1}}}} \widehat{\operatorname{PUIC}}^{\star}\left(x e^{\sigma Z_{T_{b_{1}}}}, \lambda ; K, L_{2} ; r, \delta\right)\right]=\mathbb{E}\left[e^{m Z_{T_{b_{1}}}}\left(x \mathrm{e}^{\sigma Z_{T_{b_{1}}^{-}}}\right)^{\frac{\sqrt{2 \lambda}-m}{\sigma}} f\left(\lambda ; K, L_{2} ; r, \delta\right)\right],
$$

and the result follows.

\section{Some regularity results for the Parisian option prices}

This section is devoted to the study of the regularity w.r.t. the maturity time of the Parisian option prices. These are actually computed by means of a numerical inversion of their Laplace transforms w.r.t maturity time. It is proved in Section 6 (see Proposition 6.5), that the convergence speed of the technique used to perform the numerical inversion heavily relies on the regularity of the function to be recovered.

As we will discover it, the regularity w.r.t. the maturity time of the Parisian option prices depends on the value of $b$ for single Parisian options and of $b_{1}$ and $b_{2}$ for double Parisian options, i.e. the relative positions of the initial value of the asset with the lower and upper barriers. More precisely, Theorem 5.1 states that the price of a double Parisian option is of class $\mathcal{C}^{\infty}$ when $b_{1}<0$ and $b_{2}>0$ but is discontinuous when $b_{1}>0$ or $b_{2}<0$. Concerning the special case $b_{1}=0$ or $b_{2}=0$, we state that the price of a double barrier Parisian option is continuous and may even be $\mathcal{C}^{1}$ but no more regularity can be expected. Theorem 5.1 ensues from Theorem 5.2. which deals with the regularity of the single barrier Parisian option prices. As for double Parisian options, the regularity depends on the position of $x$ compared to the barrier $L$. The proof of Theorem 5.2 is based on the regularity of the density of the "Parisian time" $T_{b}^{-}$. Section 5.2 is entirely devoted to the study of the density, when it exists, of the "Parisian times". We will prove that the r.v. $T_{b}^{-}$has a density for $b<0$, but not for $b>0$. For the special case $b=0$, we prove that $T_{0}^{-}$has a discontinuous density. 


\subsection{Regularity of option prices}

Theorem 5.1 (Regularity of double Parisian option prices). Let $f(t)$ be the "star" price of a double barrier Parisian option with maturity time $t$.

- If $b_{1}<0$ and $b_{2}>0, f$ is of class $\mathcal{C}^{\infty}$ and for all $k \geq 0, f^{(k)}(t)=\mathcal{O}\left(\mathrm{e}^{\frac{(m+\sigma)^{2}}{2} t}\right)$ when $t$ goes to infinity.

- If $b_{1}>0$ or $b_{2}<0, f$ is discontinuous in $t=D$.

- If $b_{1}=0$ or $b_{2}=0, f$ is continuous. Moreover, if $b_{1}=0$ (resp. $\left.b_{2}=0\right)$, call prices (resp. put prices) are $\mathcal{C}^{1}$ if $x \leq K$ (resp. if $x \geq K$ ).

Theorem 5.2 (Regularity of single Parisian option prices). We consider the regularity of single Parisian option prices w.r.t. maturity time.

- If $b<0$ (resp. $b>0$ ), let $f(t)$ be the price of Parisian Down (resp. Up) option with maturity $t . f$ is of class $\mathcal{C}^{\infty}$ and for all $k \geq 0, f^{(k)}(t)=\mathcal{O}\left(\mathrm{e}^{\frac{(m+\sigma)^{2}}{2} t}\right)$ when $t$ goes to infinity.

- If $b>0$ (resp. $b<0)$, the prices of Parisian Down (resp. Up) options are discontinuous in $t=D$.

- If $b=0$, Parisian option prices are continuous. Moreover, Parisian Down call (resp. Up put) prices are $\mathcal{C}^{1}$ when $x \leq k$ (resp. $x \geq k$ ).

Theorem 5.1 ensues from Proposition 3.2. Theorem 5.2 and from the following result:

Lemma 5.3. For any $b_{1}$ and $b_{2}, A\left(x, t ; K, L_{1}, L_{2} ; r, \delta\right)$ is a $\mathcal{C}^{\infty}$ function w.r.t. parameter $t$. Moreover, for all $k \geq 0, \partial_{t}^{k} A=\mathcal{O}\left(\mathrm{e}^{\frac{(m+\sigma)^{2}}{2} t}\right)$ when $t$ goes to infinity.

Proof of Theorem 5.2. The proof being the same for Down and Up options, we only prove Theorem 5.2 for Parisian Down option prices. It is sufficient to prove it for $f(t)=P D I C^{\star}(x, t ; K, L ; r, \delta)$ since $P D O C(x, t ; K, L ; r, \delta)=S C(x, T ; K ; r, \delta)-P D I C(x, t ; K, L ; r, \delta)$, the regularity of a $P D O C$ ensues from the one of a PDIC. Moreover, the following proof is still valid for Parisian Down In put prices.

Assume $b<0$. First, we prove that $f$ is a $\mathcal{C}^{\infty}$ function. Using the strong Markov property leads to

$$
f(t)=\mathbb{E}\left[e^{m Z_{T_{b}^{-}}} S C^{\star}\left(x e^{\sigma Z_{T_{b}^{-}}}, t-T_{b}^{-} ; K ; r, \delta\right) \mathbf{1}_{\left\{T_{b}^{-} \leq t\right\}}\right] .
$$

Since $Z_{T_{b}^{-}}$and $T_{b}^{-}$are independent, we get $f(t)=\mathbb{E}\left[\phi\left(t-T_{b}^{-}\right) \mathbf{1}_{\left\{T_{b}^{-} \leq t\right\}}\right]$, where $\phi(t)=$ $\mathbb{E}\left[e^{m Z_{T_{b}^{-}}} S C^{\star}\left(x e^{\sigma Z_{T_{b}^{-}}}, t ; K ; r, \delta\right)\right]$. Let $\nu$ denote the density of $Z_{T_{b}^{-}}$(see Chesnev et al. (1997) for its expression) and $p(w)=\frac{1}{\sqrt{2 \pi}} \mathrm{e}^{-\frac{w^{2}}{2}}$. We get

$$
\phi(t)=\int_{-\infty}^{\infty} d z \int_{-\infty}^{\infty} d w\left(x e^{\sigma(w+z)}-K\right)^{+} e^{m(w+z)} p(w / \sqrt{t}) \nu(z) .
$$


From this equality, we easily deduce that $\phi$ is a $\mathcal{C}^{\infty}$ function on $\mathbb{R}_{+}^{\star}$. Since $b<0$, we know from Theorem 5.4 that $T_{b}^{-}$has a density $\mu$ of class $\mathcal{C}^{\infty}$ satisfying $\mu^{(k)}(0)=\mu^{(k)}(\infty)=0$, for all $k \geq 0$. Then, we can write $f(t)=\int_{0}^{t} \phi(t-u) \mu(u) d u$ and the regularity of $\phi$ enables to complete the first part of the proof.

Second, we prove that for all $k \geq 0, f^{(k)}(t)=\mathcal{O}\left(\mathrm{e}^{\frac{(m+\sigma)^{2}}{2} t}\right)$ when $t$ goes to infinity. A change of variables in Equation (5.1) leads to

$$
f(t)=\int_{0}^{t} d \tau \int_{-\infty}^{\infty} d z \int_{-\infty}^{\infty} d w\left(x e^{\sigma(w \sqrt{\tau}+z)}-K\right)^{+} e^{m(w \sqrt{\tau}+z)} p(w) \nu(z) \mu(t-\tau) .
$$

Since $\mu$ is of class $C^{\infty}$ and all its derivatives are null at 0 and bounded on any interval $[0, T]$ (see Theorem [5.4), we get for all $k \geq 0$,

$$
f^{(k)}(t)=\int_{0}^{t} d \tau \int_{-\infty}^{\infty} d z \int_{-\infty}^{\infty} d w\left(x e^{\sigma(w \sqrt{\tau}+z)}-K\right)^{+} e^{m(w \sqrt{\tau}+z)} p(w) \nu(z) \mu^{(k)}(t-\tau) .
$$

Moreover, we can bound $f^{(k)}$

$$
\begin{aligned}
\left|f^{(k)}(t)\right| & \leq \int_{0}^{t} d \tau \int_{-\infty}^{\infty} d z \int_{-\infty}^{\infty} d w x e^{(m+\sigma)(w \sqrt{\tau}+z)} p(w) \nu(z)\left\|\mu^{(k)}\right\|_{\infty}, \\
& \leq \int_{-\infty}^{\infty} x e^{(m+\sigma) z} \nu(z) d z\left\|\mu^{(k)}\right\|_{\infty} \int_{0}^{t} e^{\frac{(m+\sigma)^{2}}{2} \tau} d \tau \\
& \leq e^{\frac{(m+\sigma)^{2}}{2} t} \frac{2 x}{(m+\sigma)^{2}}\left\|\mu^{(k)}\right\|_{\infty} \int_{-\infty}^{\infty} e^{(m+\sigma) z} \nu(z) d z .
\end{aligned}
$$

Assume $b>0$. From the definition of $f$, we know that for all $t<D, f(t)=0$, and $f(D)=\mathbb{E}\left[\phi(0) \mathbf{1}_{\left\{T_{b}^{-}=D\right\}}\right]=\mathbb{E}\left[e^{m Z_{D}}\left(x e^{\sigma Z_{D}}-K\right)_{+}\right] \mathbb{P}\left(T_{b}^{-}=D\right)$. Using Theorem $[5.4$ yields $f(D)>0$, and the result follows.

Assume $b=0$. From Theorem [5.4 we know that $T_{0}^{-}$has a density. Then, we can write, $f(t)=\int_{0}^{t} d \tau \phi(\tau-u) \mu(u) d u$. An integration by parts leads to

$$
f(t)=\phi(0) V(t)-\phi(t) V(0)+\int_{0}^{t} \phi^{\prime}(t-u) V(u) d u,
$$

where $V(u)=\mathbb{P}\left(T_{0}^{-} \leq u\right)$. Since $V$ is continuous (see the proof of Theorem 5.4) and $\phi$ is of class $\mathcal{C}^{\infty}, f$ is continuous. $f^{\prime}$ is continuous if and only if $\phi(0)=0$, i.e. if and only if $\mathbb{E}\left[e^{m Z_{T_{0}^{-}}}\left(x e^{\sigma Z_{T_{0}^{-}}}-K\right)_{+}\right]=0$, which ends the proof.

Proof of Lemma 5.3. We use the decomposition of $A \triangleq A_{1}+A_{2}$ given by Equation (3.3). We only concentrate on $A_{1}$ as $A_{2}$ can be treated in the same way. We recall that for $t \geq 0$

$$
A_{1}\left(x, t ; K, L_{1}, L_{2} ; r, \delta\right)=\mathbb{E}\left[\mathbf{1}_{\left\{T_{b_{1}}^{-} \leq t\right\}} \mathbf{1}_{\left\{T_{b_{1}}^{-} \leq T_{b_{2}}^{+}\right\}} e^{m Z_{T_{b_{1}}^{-}} P U I C^{\star}}\left(x e^{\sigma Z_{T_{b_{1}}^{-}}}, t-T_{b_{1}}^{-} ; K, L_{2} ; r, \delta\right)\right] .
$$


For the sake of simplicity, we drop all the arguments of $A$ but $t$. Since $T_{b_{1}}^{-}$is an $\mathcal{F}_{g_{t}}^{+}$-stopping time and $Z_{T_{b_{1}}^{-}}$is independent of $\mathcal{F}_{g_{T_{b_{1}}^{-}}^{+}}$, we can write

$$
A_{1}(t)=\mathbb{E}\left[\mathbf{1}_{\left\{T_{b_{1}}^{-} \leq t\right\}} \mathbf{1}_{\left\{T_{b_{1}}^{-} \leq T_{b_{2}}^{+}\right\}} \mathbb{E}\left[e^{m Z_{T_{b_{1}}}} P U I C^{\star}\left(x e^{\sigma Z_{T_{b_{1}}}^{-}}, t-u ; K, L_{2} ; r, \delta\right)\right]_{\left.\right|_{u=T_{b_{1}}}}\right] \text {. }
$$

Note that $x e^{\sigma Z_{T_{b_{1}}}}<L_{1}$. It is fairly easy to adapt the proof of Theorem 5.2 in the case $b<0$ to prove that $f(t)=\mathbb{E}\left[e^{m Z_{T_{b_{1}}}^{-}} P U I C^{\star}\left(x e^{\sigma Z_{T_{b_{1}}}}, t ; K, L_{2} ; r, \delta\right)\right]$ is of class $\mathcal{C}^{\infty}$ for $t>0$ and that $f^{(k)}(t)=\mathcal{O}\left(\mathrm{e}^{\frac{(m+\sigma)^{2}}{2} t}\right)$. Moreover $f(t)=0$ if $t<D$. So $\bar{f}(t)=\mathbf{1}_{\{t \geq 0\}} f(t)$ is of class $\mathcal{C}^{\infty}$ on $\mathbb{R}$. Then, Equation (5.2) becomes

$$
A_{1}(t)=\mathbb{E}\left[\mathbf{1}_{\left\{T_{b_{1}}^{-} \leq T_{b_{2}}^{+}\right\}} \bar{f}\left(t-T_{b_{1}}^{-}\right)\right] .
$$

Because $\bar{f}$ is of class $\mathcal{C}^{\infty}$ on $\mathbb{R}$ and $T_{b_{1}}^{-}$is a positive random variable, it is obvious that $A_{1}$ is of class $\mathcal{C}^{\infty}$ on $\mathbb{R}^{+}$by repeatedly applying the bounded convergence theorem. Moreover for any $k \geq 0$,

$$
\partial_{t}^{k} A_{1}(t)=\mathbb{E}\left[\mathbf{1}_{\left\{T_{b_{1}}^{-} \leq T_{b_{2}}^{+}\right\}} \bar{f}^{(k)}\left(t-T_{b_{1}}^{-}\right)\right] \quad \text { and } \quad\left|\partial_{t}^{k} A_{1}(t)\right| \leq \mathbb{E}\left[\left|\bar{f}^{(k)}\left(t-T_{b_{1}}^{-}\right)\right|\right] .
$$

The fact that $\partial_{t}^{k} A_{1}(t)=\mathcal{O}\left(\mathrm{e}^{\frac{(m+\sigma)^{2}}{2} t}\right)$ directly ensues from what has been said above.

\subsection{Regularity of the density of the "Parisian times" $T_{b}^{-}$and $T_{b}^{+}$}

In this section, $T_{b}^{-}$and $T_{b}^{+}$are related to a Brownian motion $W$. We recall (see Definition 2.5) that $T_{b}^{-}(W)=\inf \left\{t>0:\left(t-g_{t}^{b}(W)\right) \mathbf{1}_{\left\{W_{t}<b\right\}} \geq D\right\}$.

Theorem 5.4. The following assertions hold

- For $b<0$ (resp. $b>0$ ), the r.v. $T_{b}^{-}$(resp. $\left.T_{b}^{+}\right)$has a density $\mu$ w.r.t Lebesgue's measure. $\mu$ is of class $C^{\infty}$ and for all $k \geq 0, \mu^{(k)}(0)=\mu^{(k)}(\infty)=0$.

- For $b>0$ (resp. $b<0)$, the r.v. $T_{b}^{-}$(resp. $\left.T_{b}^{+}\right)$is not absolutely continuous w.r.t Lebesgue's measure and $\mathbb{P}\left(T_{b}^{-}=D\right)>0$ (resp. $\left.\mathbb{P}\left(T_{b}^{+}=D\right)>0\right)$.

- $T_{0}^{-}$has a density which tends to infinity in $D^{+}$and equals 0 in $D^{-}$. Nonetheless, the jump in $D$ is integrable.

One can refer to Figure 2 to have an overview of what the density and cumulative distribution of $T_{0}^{-}$look like. These functions are computed by numerically inverting the Laplace transform of $T_{0}^{-}$. To prove the first part of this proposition, we need the two following lemmas.

Lemma 5.5. We define

$$
\mathcal{N}(x+i y)=\frac{1}{\sqrt{2 \pi}} \int_{-\infty}^{x} \mathrm{e}^{-\frac{(v+i y)^{2}}{2}} d v
$$


Then, $\mathcal{N}$ is the analytic prolongation of the cumulative normal distribution function on the complex plane. Moreover, the following equivalent holds

$$
\mathcal{N}(r(1+i)) \sim 1 \text { when } r \rightarrow \infty .
$$

Lemma 5.6. For $b<0$, we have for $u \in \mathbb{R}$

$$
\mathbb{E}\left(\mathrm{e}^{-i u T_{b}^{-}}\right)=\mathcal{O}\left(\mathrm{e}^{-|b| \sqrt{|u|}}\right) \text { when }|u| \rightarrow \infty .
$$

The proofs of Lemmas [5.5 and [5.6 are postponed to Appendix $\mathrm{A}$

Proof of Theorem 5.4. We only prove Theorem 5.4 for the r.v. $T_{b}^{-}$.

Assume $b<0$. We recall that (see Appendix $[$ for more details)

$$
\mathbb{E}\left(\mathrm{e}^{-\frac{\lambda^{2}}{2} T_{b}^{-}}\right)=\frac{\mathrm{e}^{\lambda b}}{\psi(\lambda \sqrt{D})}
$$

We define $\mathcal{O}=\left\{z \in \mathbb{C} ;-\frac{\pi}{4}<\arg (z)<\frac{\pi}{4}\right\}$. One can easily prove that the function $z \longmapsto$ $\mathbb{E}\left(\mathrm{e}^{-\frac{z^{2}}{2} T_{b}^{-}}\right)$is holomorphic on the open set $\mathcal{O}$ and hence analytic. Moreover, $z \longmapsto \frac{\mathrm{e}^{z b}}{\psi(z \sqrt{D})}$ is also analytic on $\mathcal{O}$ except perhaps in a countable number of isolated points. These two functions coincide on $\mathbb{R}^{+}$, so they are equal on $\mathcal{O}$. Consequently, we can derive the following equality. For all $z \in \mathbb{C}$ with positive real part, we have

$$
\mathbb{E}\left(\mathrm{e}^{-z T_{b}^{-}}\right)=\frac{\mathrm{e}^{\sqrt{2 z} b}}{\psi(\sqrt{2 z D})}
$$

We use the following convention: for any $z \in \mathbb{C}$ with positive real part, $\sqrt{z}$ is the only complex number $z^{\prime} \in \mathcal{O}$ such that $z=z^{\prime} z^{\prime}$.

Thanks to the continuity of both terms in (5.4), the equality also holds for pure imaginary numbers. Hence, by setting $z=i u$ for $u \in \mathbb{R}$ in Equation (5.4), we obtain the Fourier transform of $T_{b}^{-}$

$$
\mathbb{E}\left(\mathrm{e}^{-i u T_{b}^{-}}\right)=\frac{\mathrm{e}^{\sqrt{2 u i} b}}{\psi(\sqrt{2 i u D})} .
$$

From Lemma [5.6] we know that the Fourier transform of $T_{b}^{-}$is integrable on $\mathbb{R}$, thus the r.v. $T_{b}^{-}$has a density $\mu$ w.r.t. the Lebesgue measure given by

$$
\mu(t)=\frac{1}{2 \pi} \int_{-\infty}^{\infty} \frac{\mathrm{e}^{\sqrt{2 u i b}}}{\psi(\sqrt{2 i u D})} \mathrm{e}^{-i u t} d u
$$

Moreover, thanks to Lemma [5.6] $u \longmapsto u^{k} \frac{\mathrm{e}^{\sqrt{2 u i b}}}{\psi(\sqrt{2 i u D})}$ is integrable and continuous. Hence, $\mu$ is of class $C^{\infty}$. Since $\mu(t)=0$ for $t<D$, for all $k \geq 0, \mu^{(k)}(0)=0$. Lemma A.1 yields that for all $k \geq 0, \lim _{t \rightarrow \infty} \mu^{(k)}(t)=0$.

Assume $b>0 . \mathbb{P}\left(T_{b}^{-}=D\right)=\mathbb{P}\left(\sup _{0 \leq u \leq D} W_{u} \leq b\right)$, which yields the result. 
Assume $b=0$. First, let us prove that $T_{0}^{-}$has a density. From Chesnev et al. (1997), we know that the Fourier transform of $T_{0}^{-}$is given by

$$
\phi(u)=\mathbb{E}\left[\mathrm{e}^{-i u T_{0}^{-}}\right]=\frac{1}{\psi(\sqrt{2 i u D})} .
$$

Using Lemma [5.5] $|\psi(u)| \underset{|u| \rightarrow \infty}{\sim} 2 \sqrt{\pi D|u|}$. So, $\frac{|\psi(u)|}{u}$ is integrable. Hence, the cumulative distribution function $F(t)=\mathbb{P}\left(T_{0}^{-} \leq t\right)$ is absolutely continuous w.r.t. Lebesgue's measure (see Abate and Whitt (1992) for more details). Henceforth, the r.v. $T_{0}^{-}$has a density.

We will now prove that the density of $T_{0}^{-}$is discontinuous in $D$. Let $V(t)$ denote the length of the longest excursion of the Brownian motion up to time $t$. From the scaling property of the Brownian motion, it is clear that $V(t)$ and $t V(1)$ are equal in distribution. In the sequel, we denote $V(1)$ by $V$. We can $\operatorname{link} V$ and $F$. For $t \geq D$, we have

$$
F(t)=\mathbb{P}\left(T_{0}^{-} \leq t\right)=\mathbb{P}(V(t) \geq D)=\mathbb{P}\left(V \geq \frac{D}{t}\right) .
$$

From Pitman and Yor (1997), we know that $V$ has the same distribution as $\frac{1}{1+R_{1}+R_{1} R_{2}+R_{1} R_{2} R_{3}+\ldots}$ where $R_{n}$ has the $\beta\left(\frac{n}{2}, 1\right)$ distribution and the $R_{n}$ are mutually independent. We are interested in the limit of $\frac{F(D+\varepsilon D)-F(D)}{\varepsilon D}$ when $\varepsilon$ tends to zero.

$$
F(D+\varepsilon D)-F(D)=\mathbb{P}\left(V \geq \frac{1}{1+\varepsilon}\right)=\mathbb{P}\left(R_{1}+R_{1} R_{2}+R_{1} R_{2} R_{3}+\cdots \leq \varepsilon\right) .
$$

We have the following inclusions

$$
\left(\left\{R_{1} \leq \frac{\varepsilon}{2}\right\} \cup\left\{1+R_{2}+R_{2} R_{3}+\cdots \leq 2\right\}\right) \subset\left\{R_{1}+R_{1} R_{2}+\cdots \leq \varepsilon\right\} \subset\left\{R_{1} \leq \varepsilon\right\} .
$$

Moreover, $\mathbb{P}\left(R_{1} \leq \frac{\varepsilon}{2}, 1+R_{2}+R_{2} R_{3}+\cdots \leq 2\right)=\mathbb{P}\left(R_{1} \leq \frac{\varepsilon}{2}\right) \mathbb{P}\left(R_{2}+R_{2} R_{3}+\cdots \leq 1\right)$. From the equality $\mathbb{P}\left(R_{1} \leq \frac{\varepsilon}{2}\right)=\sqrt{\frac{\varepsilon}{2}}$, we deduce using Equation (5.6) that

$$
c \sqrt{\varepsilon} \leq \mathbb{P}\left(V \geq \frac{1}{1+\varepsilon}\right) \leq \sqrt{\varepsilon},
$$

where $0<c \leq \frac{1}{\sqrt{2}}$. Plugging this into Equation (5.5), proves that $\frac{F(D+\varepsilon D)-F(D)}{\varepsilon D}$ goes to infinity at the rate $\frac{1}{\sqrt{\varepsilon}}$ when $\varepsilon$ goes to zero. Note that the jump is however integrable. Hence, the density of $T_{0}^{-}$is not differentiable in $D$.

\section{The inversion of Laplace transforms}

This section is devoted to the numerical inversion of the Laplace transforms computed previously. We recall that the Laplace transforms are computed with respect to the maturity time. We explain how to recover a function from its Laplace transform using a contour integral. The real problem is how to numerically evaluate this complex integral. This is done in two separate steps involving two different errors. First, as explained in Section 6.2 we replace the integral by a series. The first step creates a discretisation error, which is handled by Proposition 6.3. Secondly, one has to compute a non-finite series. This can be achieved by simply truncating the series but it leads to a tremendously slow convergence. Here, we prefer to use the Euler acceleration as presented in Section 6.3 Proposition 6.5 states an upper-bound for the error due to the accelerated computation of the non finite series. Theorem 6.6 gives a bound for the global error. 


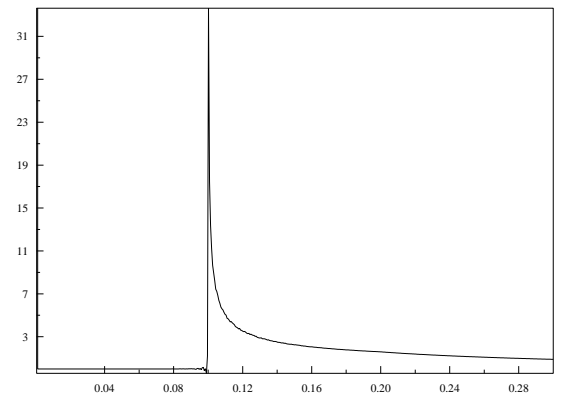

(a)

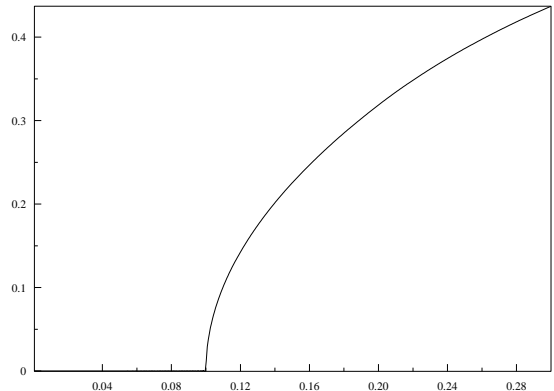

(b)

Figure 2: Parisian time $T_{0}^{-}$: (a) the density function; (b) the cumulative distribution function.

\subsection{Analytical prolongations}

Because the Laplace inversion is performed in the complex plane, we have to extend the expressions obtained for the Laplace transforms to the complex plane. To do so, we use Lemma 5.5 which gives the analytic prolongation of the normal cumulative distribution function on the complex plane. From Proposition 6.1] it is quite easy to show that the expressions obtained for a real value of the Laplace parameter are still valid for a complex one with real part larger than $\frac{(m+\sigma)^{2}}{2}$.

Proposition 6.1 (abscissa of convergence). The abscissa of convergence of the Laplace transforms of the star prices of Parisian options is smaller than $\frac{(m+\sigma)^{2}}{2}$. All these Laplace transforms are analytic on the complex half plane $\left\{z \in \mathbb{C}: \mathcal{R e}(z)>\frac{(m+\sigma)^{2}}{2}\right\}$.

Proof. It is sufficient to notice that the star price of a Parisian option is bounded by $\mathbb{E}\left(\mathrm{e}^{m Z_{T}}\left(x \mathrm{e}^{\sigma W_{T}}+K\right)\right)$.

$$
\mathbb{E}\left(\mathrm{e}^{m Z_{T}}\left(x \mathrm{e}^{\sigma W_{T}}+K\right)\right) \leq K \mathrm{e}^{\frac{m^{2}}{2} T}+x \mathrm{e}^{\frac{(m+\sigma)^{2}}{2} T}=\mathcal{O}\left(\mathrm{e}^{\frac{(m+\sigma)^{2}}{2} T}\right) .
$$

Hence, Widder (1941, Theorem 2.1) yields that the abscissa of convergence of the Laplace transforms of the star prices is smaller that $\frac{(m+\sigma)^{2}}{2}$. The second part of the proposition ensues from Widder (1941, Theorem 5.a).

With the definition of $\mathcal{N}$ given by Equation (5.3), it is clear that all the expressions obtained so far for the Laplace transforms are also valid for complex values of $\lambda$ satisfying $\mathcal{R e}(\lambda)>\frac{(m+\sigma)^{2}}{2}$ since their are analytic on the complex half plane $\left\{z \in \mathbb{C}: \mathcal{R e}(z)>\frac{(m+\sigma)^{2}}{2}\right\}$.

\subsection{The Fourier series representation}

Thanks to Widder 1941, Theorem 9.2), we know how to recover a function from its Laplace transform. 
Theorem 6.2. Let $f$ be a continuous function defined on $\mathbb{R}^{+}$and $\alpha$ a positive number. If the function $f(t) \mathrm{e}^{-\alpha t}$ is integrable, then given the Laplace transform $\hat{f}, f$ can be recovered from the contour integral

$$
f(t)=\frac{1}{2 \pi i} \int_{\alpha-i \infty}^{\alpha+i \infty} \mathrm{e}^{s t} \hat{f}(s) d s, \quad t>0 .
$$

The variable $\alpha$ has to be chosen greater than the abscissa of convergence of $\hat{f}$. The abscissa of convergence of the Laplace transforms of the double barrier Parisian option prices computed previously is smaller than $(m+\sigma)^{2} / 2$ (the Laplace transforms of the single barrier Parisian options - recalled in Appendix D- are given for $\left.\lambda>\frac{(m+\sigma)^{2}}{2}\right)$. Hence, $\alpha$ must be chosen strictly greater than $(m+\sigma)^{2} / 2$.

For any real valued function satisfying the hypotheses of Theorem 6.2 we introduce a trapezoidal discretisation of Equation (6.1)

$$
f_{\pi / t}(t)=\frac{e^{\alpha t}}{2 t} \widehat{f}(\alpha)+\frac{e^{\alpha t}}{t} \sum_{k=1}^{\infty}(-1)^{k} \mathcal{R e}\left(\widehat{f}\left(\alpha+i \frac{k \pi}{t}\right)\right) .
$$

Proposition 6.3. If $f$ is a continuous bounded function satisfying $f(t)=0$ for $t<0$, we have

$$
\left|e_{\pi / t}(t)\right|=\left|f(t)-f_{\pi / t}(t)\right| \leq\|f\|_{\infty} \frac{e^{-2 \alpha t}}{1-e^{-2 \alpha t}} .
$$

Remark 6.4. For the upper bound in Proposition 6.3 to be smaller than $10^{-8}\|f\|_{\infty}$, one has to choose $2 \alpha t=18.4$. In fact, this bound holds for any choice of the discretisation step $h$ satisfying $h<2 \pi / t$.

Simply truncating the summation in the definition of $f_{\pi / t}$ to compute the trapezoidal integral is far too rough to provide a fast and accurate numerical inversion. One way to improve the convergence of the series is to use the Euler summation.

\subsection{The Euler summation}

To improve the convergence of a series $S$, we use the Euler summation technique as described by Abate et al. (1999), which consists in computing the binomial average of $q$ terms from the $p$-th term of the series $S$. The binomial average obviously converges to $S$ as $p$ goes to infinity. The following proposition describes the convergence rate of the binomial average to the infinite series $f_{\pi / t}(t)$ when $p$ goes to $\infty$.

Proposition 6.5. Let $f$ be a function of class $\mathcal{C}^{q+4}$ such that there exists $\epsilon>0$ s.t. $\forall k \leq$ $q+4, f^{(k)}(s)=\mathcal{O}\left(e^{(\alpha-\epsilon) s}\right)$. We define $s_{p}(t)$ as the approximation of $f_{\pi / t}(t)$ when truncating the non-finite series in (6.2) to $p$ terms

$$
s_{p}(t)=\frac{e^{\alpha t}}{2 t} \widehat{f}(\alpha)+\frac{e^{\alpha t}}{t} \sum_{k=1}^{p}(-1)^{k} \mathcal{R e}\left(\widehat{f}\left(\alpha+i \frac{\pi k}{t}\right)\right),
$$

and $E(q, p, t)=\sum_{k=0}^{q} C_{q}^{k} 2^{-q} s_{p+k}(t)$. Then,

$$
\left|f_{\pi / t}(t)-E(q, p, t)\right| \leq \frac{t e^{\alpha t}\left|f^{\prime}(0)-\alpha f(0)\right|}{\pi^{2}} \frac{p !(q+1) !}{2^{q}(p+q+2) !}+\mathcal{O}\left(\frac{1}{p^{q+3}}\right)
$$

when $p$ goes to infinity. 
Using Propositions 6.3 and 6.5 we get the following result concerning the global error on the numerical computation of the price of a double barrier Parisian call option

Theorem 6.6. Let $f$ be the price of a double barrier Parisian call option. Using the notations of Proposition 6.5, we have

$$
|f(t)-E(q, p, t)| \leq S_{0} \frac{e^{-2 \alpha t}}{1-e^{-2 \alpha t}}+\frac{e^{\alpha t} t\left|f^{\prime}(0)-\alpha f(0)\right| p !(q+1) !}{\pi^{2} 2^{q}(p+q+2) !}+\mathcal{O}\left(\frac{1}{p^{q+3}}\right)
$$

where $\alpha$ is defined in Theorem 6.2

Proof of Theorem [6.6. $f$ being the price of a double barrier Parisian call option, we know that $f$ is bounded by $S_{0}$. Moreover, $f$ is continuous (actually of class $\mathcal{C}^{\infty}$, see Appendix 5.1). Hence, Proposition 6.3 yields the first term on the right-hand side of (6.4).

Relying on Theorem 5.1] we know that $f^{\star}$ is of class $\mathcal{C}^{\infty}$ and $\left(f^{\star}\right)^{(k)}(t)=\mathcal{O}\left(e^{\frac{(m+\sigma)^{2}}{2} t}\right), \forall k \geq 0$. Since $f(t)=\mathrm{e}^{-\left(r+m^{2} / 2\right) t} f^{\star}(t)$, it is quite obvious that $f$ is also of class $\mathcal{C}^{\infty}$ and $f^{(k)}(t)=$ $\mathcal{O}\left(\mathrm{e}^{\left((m+\sigma)^{2} / 2-\left(r+m^{2} / 2\right)\right) t}\right), \forall k \geq 0$. Since $\alpha>\frac{(m+\sigma)^{2}}{2}$, we apply Proposition 6.5 to get the result.

Proof of Proposition [6.5. We compute the difference between two successive terms.

$$
E(q, p+1, t)-E(q, p, t)=\frac{\mathrm{e}^{\alpha t}}{2^{q} t} \sum_{k=0}^{q} C_{q}^{k}(-1)^{p+1+k} a_{p+k+1},
$$

where

$$
a_{p}=\int_{0}^{+\infty} e^{-\alpha s} \cos \left(\frac{p}{t} \pi s\right) f(s) d s .
$$

Let $g(s)=e^{-\alpha s} f(s)$. Since $g^{(k)}(\infty)=0$ for $k \leq q+3$ and $g^{(q+4)}$ is integrable, we can perform $(q+3)$ integrations by parts in (6.5) to obtain a Taylor expansion when $p$ goes to infinity

$$
a_{p}=\frac{c_{2}}{p^{2}}+\frac{c_{4}}{p^{4}}+\cdots+\frac{c_{q}}{p^{2[(q+3) / 2]}}+\mathcal{O}\left(\frac{1}{p^{q+4}}\right)
$$

with $c_{2}=\frac{t^{2}\left(f^{\prime}(0)-\alpha f(0)\right)}{\pi^{2}}$.

We can rewrite [6.6)

$$
a_{p}=\frac{c_{2}}{p(p+1)}+\frac{c_{3}^{\prime}}{p(p+1)(p+2)}+\cdots+\frac{c_{q+3}^{\prime}}{p(p+1) \cdots(p+q+2)}+\mathcal{O}\left(\frac{1}{p^{q+4}}\right) .
$$

Some elementary computations show that for $j \geq 2$

$$
\sum_{k=0}^{q} C_{q}^{k}(-1)^{p+1+k} \frac{1}{(p+k+1) \cdots(p+k+j)}=(-1)^{p+1} \frac{p !(q+j-1) !}{(j-1) !(p+q+j) !} .
$$

Computing $\sum_{k=0}^{q} C_{q}^{k}(-1)^{p+1+k} a_{p+k+1}$ leads to

$$
E(q, p+1, t)-E(q, p, t)=(-1)^{p+1} \frac{\mathrm{e}^{\alpha t}}{2^{q t}}\left\{c_{2} \frac{p !(q+1) !}{(p+q+2) !}-c_{3}^{\prime} \frac{(p+1) !(q+2) !}{2(p+q+4) !}\right\}+\mathcal{O}\left(\frac{1}{p^{q+4}}\right) .
$$


Moreover, $\frac{p !(q+1) !}{(p+q+2) !}$ and $\frac{(p+1) !(q+2) !}{(p+q+4) !}$ are both decreasing w.r.t $p$, so

$$
|E(q, \infty, t)-E(q, p, t)| \leq\left|c_{2}\right| \frac{\mathrm{e}^{\alpha t}}{2^{q} t} \frac{p !(q+1) !}{(p+q+2) !}+\mathcal{O}\left(\frac{1}{p^{q+3}}\right) .
$$

Remark 6.7. Whereas Proposition 6.3 in fact holds for any $h<2 \pi / t$, the proof of Proposition [6.5] is essentially based on the choice of $h=\pi / t$ since the key point is to be able to write $E(q, p+1, t)-E(q, p, t)$ as the general term of an alternating series. The impressive convergence rate of $E(q, p, t)$ definitely relies on the choice of this particular discretisation step. For a general step $h$, it is much more difficult to study the convergence rate and one cannot give an explicit upper bound.

Remark 6.8. For $2 \alpha t=18.4$ and $q=p=15$, the global error is bounded by $S_{0} 10^{-8}+$ $t\left|f^{\prime}(0)-\alpha f(0)\right| 10^{-11}$. As one can see, the method we use to invert Laplace transforms provides a very good accuracy with few computations.

Remark 6.9. Considering the case of call options in Theorem 6.6] is sufficient since put prices are computed using parity relations and their accuracy is hung up to the one of call prices. Theorem 6.6 also holds for single barrier Parisian options.

\section{A Proof of Lemmas 5.5 and 5.6}

Proof of Lemma 5.5

$$
\mathcal{N}(x+i y)=\frac{1}{\sqrt{2 \pi}} \int_{-\infty}^{x} \mathrm{e}^{-\frac{(v+i y)^{2}}{2}} d v
$$

It is easy to check that $\partial_{x} \mathcal{N}(x+i y)-\partial_{y} \mathcal{N}(x+i y)=0$ and this definition coincides with the cumulative normal distribution function on the real axis, so it is the unique analytic prolongation. We write $\mathcal{N}(x+i y)=\mathcal{N}(x)+\int_{0}^{y} \partial_{r} \mathcal{N}(x+i r) d r$, to get

$$
\begin{aligned}
\mathcal{N}(x+i y) & =\mathcal{N}(x)-i \frac{1}{\sqrt{2 \pi}} \int_{0}^{y} \int_{-\infty}^{x}(v+i u) \mathrm{e}^{-\frac{(v+i u)^{2}}{2}} d v d u, \\
& =\mathcal{N}(x)+i \frac{1}{\sqrt{2 \pi}} \int_{0}^{y} \mathrm{e}^{-\frac{(x+i u)^{2}}{2}} d u .
\end{aligned}
$$

Taking $x+i y=r(1+i)$ gives

$$
\begin{aligned}
\mathcal{N}(r(1+i)) & =\mathcal{N}(r)+i \frac{1}{\sqrt{2 \pi}} \int_{0}^{r} \mathrm{e}^{-\frac{(r+i u)^{2}}{2}} d u, \\
& =\mathcal{N}(r)+i \frac{1}{\sqrt{2 \pi}} \int_{0}^{1} \mathrm{e}^{\frac{r^{2}}{2}\left(t^{2}-1\right)} \mathrm{e}^{-i t r^{2}} r d t .
\end{aligned}
$$

For $t \in[0,1), \mathrm{e}^{\frac{r^{2}}{2}\left(t^{2}-1\right)} r$ tends to 0 when $r$ goes to infinity. The function $r \longmapsto \mathrm{e}^{\frac{r^{2}}{2}\left(t^{2}-1\right)} r$ is maximum for $r=\frac{1}{1-t^{2}}$, hence the following upper bound holds

$$
\mathrm{e}^{\frac{r^{2}}{2}\left(t^{2}-1\right)} r \leq \frac{1}{1-t^{2}} \mathrm{e}^{\frac{1}{2\left(t^{2}-1\right)}} \quad \text { for all } t \in[0,1) .
$$

The upper bound is integrable on $[0,1)$, so by using the bounded convergence theorem, we can assert that the integral on the right hand side of (A.1) tends to 0 when $r$ goes to infinity. 
Proof of Lemma 5.6. We only do the proof for $u>0$. For $r>0$,

$$
\psi(r(1+i))=1+r(1+i) \sqrt{2 \pi} \mathrm{e}^{r^{2} i} \mathcal{N}(r(1+i)) .
$$

Using the equivalent of $\mathcal{N}(r(1+i))$ when $r$ goes to infinity (see Lemma 5.5) enables to establish that $|\psi(r(1+i))| \sim 2 r \sqrt{\pi}$ when $r$ goes to infinity. Noticing that $\sqrt{i u}=\frac{\sqrt{2 u}}{2}(1+i)$ ends the proof.

Here is a quite obvious lemma we used in the proof of Theorem 5.4.

Lemma A.1. Let $g$ be an integrable function on $\mathbb{R}$, then

$$
\lim _{t \rightarrow \infty} \int_{-\infty}^{\infty} g(u) \mathrm{e}^{i u t} d u=0 .
$$

\section{B Proof of Proposition 6.3}

To prove Proposition 6.3, we need the following result adapted from Abate et al. 1999, Theorem 5).

Lemma B.1. For any continuous and bounded function $f$ such that $f(t)=0$ for $t<0$, we have

$$
e_{\pi / t}(t)=f_{\pi / t}(t)-f(t)=\sum_{\substack{k=-\infty \\ k \neq 0}}^{\infty} f(t(1+2 k)) \mathrm{e}^{-2 k \alpha t} .
$$

Proof of Proposition 6.3, By performing a change of variables $s=\alpha+i u$ in the integral in (6.1), we can easily obtain an integral of a real variable.

$$
f(t)=\frac{e^{\alpha t}}{2 \pi} \int_{-\infty}^{+\infty} \widehat{f}(\alpha+i u)(\cos (u t)+i \sin (u t)) d u .
$$

Moreover, since $f$ is a real valued function, the imaginary part of the integral vanishes

$$
\left.f(t)=\frac{e^{\alpha t}}{2 \pi} \int_{-\infty}^{+\infty} \mathcal{R e}(\widehat{f}(\alpha+i u)) \cos (u t)-\mathcal{I} \mathrm{m}(\widehat{f}(\alpha+i u)) \sin (u t)\right) d u .
$$

We notice that

$$
\mathcal{I m}(\widehat{f}(\alpha+i u))=-\mathcal{I} \mathrm{m}(\widehat{f}(\alpha-i u)), \quad \mathcal{R} \mathrm{e}(\widehat{f}(\alpha+i u))=\mathcal{R e}(\widehat{f}(\alpha-i u)) .
$$

So,

$$
\left.f(t)=\frac{e^{\alpha t}}{\pi} \int_{0}^{+\infty} \mathcal{R e}(\widehat{f}(\alpha+i u)) \cos (u t)-\mathcal{I} \mathrm{m}(\widehat{f}(\alpha+i u)) \sin (u t)\right) d u .
$$

Using a trapezoidal integral with a step $h=\frac{\pi}{t}$ leads to Equation (6.2). Remembering that $f(t)=0$ for $t<0$, we can easily deduce from Lemma B.1 that

$$
e_{\pi / t}(t)=\sum_{k=1}^{\infty} f(t(1+2 k)) \mathrm{e}^{-2 k \alpha t} .
$$

Taking the upper bound of $f$ yields (6.3). 


\section{Laplace transforms}

First, we define the function $\psi$, strongly related to the cumulative normal distribution $\mathcal{N}$.

Definition C.1 (Definition of $\psi$ ). Let $z$ be in $\mathbb{R}$.

$$
\psi(z)=\int_{0}^{\infty} x \exp \left(-\frac{x^{2}}{2}+z x\right) d x=1+z \sqrt{2 \pi} \exp \left(\frac{z^{2}}{2}\right) \mathcal{N}(z)
$$

C.1 Laplace transforms for $T_{b}^{-}, T_{b}^{+}, Z_{T_{b}^{-}}$and $Z_{T_{b}^{+}}$

The following Lemma ensues from Chesnev et al. (1997, Sections 8.3 and 8.4), giving the Laplace transforms of $T_{b}^{-}$and $T_{b}^{+}$, for $b \in \mathbb{R}$.

Lemma C.2. Let $\theta$ denote $\sqrt{2 \lambda}$. We recall that the function $\psi$ is given in Definition C.1. Then,

$$
\begin{aligned}
\mathbb{E}\left[e^{-\lambda T_{b}^{-}}\right] & =\frac{e^{\theta b}}{\psi(\theta \sqrt{D})} \quad \text { for } b<0 \\
& =e^{-\lambda D}\left(1-2 \mathcal{N}\left(-\frac{b}{\sqrt{D}}\right)\right) \\
& +\psi(\theta \sqrt{D})^{-1}\left[e^{-\theta b} \mathcal{N}\left(\theta \sqrt{D}-\frac{b}{\sqrt{D}}\right)+e^{\theta b} \mathcal{N}\left(-\theta \sqrt{D}-\frac{b}{\sqrt{D}}\right)\right] \text { otherwise. } \\
\mathbb{E}\left[e^{-\lambda T_{b}^{+}}\right] & =\frac{e^{-\theta b}}{\psi(\theta \sqrt{D})} \quad \text { for } b>0 \\
& =e^{-\lambda D}\left(1-2 \mathcal{N}\left(\frac{b}{\sqrt{D}}\right)\right) \\
& +\psi(\theta \sqrt{D})^{-1}\left[e^{\theta b} \mathcal{N}\left(\theta \sqrt{D}+\frac{b}{\sqrt{D}}\right)+e^{-\theta b} \mathcal{N}\left(-\theta \sqrt{D}+\frac{b}{\sqrt{D}}\right)\right] \text { otherwise. }
\end{aligned}
$$

To compute the Laplace transforms of $Z_{T^{-}}$and $Z_{T_{b}^{+}}$we use the densities of these random variables. We refer to Chesnev et al. (1997, Sections 8.3 and 8.4) for an expression of these densities (note that there is a misprint in Chesnev et al. (1997, Appendix 8.3): indicator $\mathbf{1}_{\{x<b\}}$ is missing in the formula of $\mathbb{P}\left(Z_{D} \in d x, T_{b}>D\right)$ case $\left.b>0\right)$.

Lemma C.3. Let $\theta$ denote $\sqrt{2 \lambda}$. We recall that the function $\psi$ is given in Definition C.1 Then,

$$
\begin{aligned}
\mathbb{E}\left[e^{-\theta Z_{T_{b}^{-}}}\right] & =e^{-\theta b} \psi(\theta \sqrt{D}) \quad \text { for } b<0 \\
& =2 \mathcal{N}\left(-\frac{b}{\sqrt{D}}\right) e^{-\theta b} \psi(\theta \sqrt{D}) \\
& +e^{\lambda D}\left[\mathcal{N}\left(\frac{b}{\sqrt{D}}+\theta \sqrt{D}\right)-e^{-2 \theta b} \mathcal{N}\left(-\frac{b}{\sqrt{D}}+\theta \sqrt{D}\right)\right] \text { otherwise. }
\end{aligned}
$$




$$
\begin{aligned}
\mathbb{E}\left[e^{-\theta Z_{T_{b}^{+}}}\right] & =e^{-\theta b} \psi(-\theta \sqrt{D}) \quad \text { for } b>0 \\
& =2 \mathcal{N}\left(\frac{b}{\sqrt{D}}\right) e^{-\theta b} \psi(-\theta \sqrt{D}) \\
& +e^{\lambda D}\left[\mathcal{N}\left(-\frac{b}{\sqrt{D}}-\theta \sqrt{D}\right)-e^{-2 \theta b} \mathcal{N}\left(\frac{b}{\sqrt{D}}-\theta \sqrt{D}\right)\right] \text { otherwise }
\end{aligned}
$$

\section{C.2 Formulas for $\mathbb{E}\left[e^{-\lambda T_{b_{1}}^{-}} \mathbf{1}_{\left\{T_{b_{1}}^{-}<T_{b_{2}}^{+}\right\}}\right]$and $\mathbb{E}\left[e^{-\lambda T_{b_{2}}^{+}} \mathbf{1}_{\left\{T_{b_{2}}^{+}<T_{b_{1}}^{-}\right\}}\right]$}

Lemma C.4. Let $\theta$ denote $\sqrt{2 \lambda}$. We recall that the function $\psi$ is given in Definition C.1. Then,

$$
\begin{aligned}
& \mathbb{E}\left[e^{-\lambda T_{b_{1}}^{-}} \mathbf{1}_{\left\{T_{b_{1}}^{-}<T_{b_{2}}^{+}\right\}}\right]=\frac{\mathbb{E}\left[e^{-\lambda T_{b_{1}}^{-}}\right]-a_{1} \mathbb{E}\left[e^{-\lambda T_{b_{2}}^{+}}\right]}{1-a_{1} a_{2}}, \\
& \mathbb{E}\left[e^{-\lambda T_{b_{2}}^{+}} \mathbf{1}_{\left\{T_{b_{2}}^{+}<T_{b_{1}}^{-}\right\}}\right]=\frac{\mathbb{E}\left[e^{-\lambda T_{b_{2}}^{+}}\right]-a_{2} \mathbb{E}\left[e^{-\lambda T_{b_{1}}^{-}}\right]}{1-a_{1} a_{2}},
\end{aligned}
$$

where $a_{1}=\frac{e^{\theta b_{1}}}{\psi(\theta \sqrt{D})} \mathbb{E}\left[\exp \left(-\theta Z_{T_{b_{2}}^{+}}\right)\right]$and $a_{2}=\frac{e^{-\theta b_{2}}}{\psi(\theta \sqrt{D})} \mathbb{E}\left[\exp \left(\theta Z_{T_{b_{1}}^{-}}\right)\right]$.

Proof. Let $E_{-}$(resp. $\left.E_{+}\right)$denote $\mathbb{E}\left[e^{-\lambda T_{b_{1}}^{-}} \mathbf{1}_{\left\{T_{b_{1}}^{-}<T_{b_{2}}^{+}\right\}}\right]$(resp. $\left.\mathbb{E}\left[e^{-\lambda T_{b_{2}}^{+}} \mathbf{1}_{\left\{T_{b_{2}}^{+}<T_{b_{1}}^{-}\right\}}\right]\right)$. Then,

$$
\begin{aligned}
& \mathbb{E}\left[e^{-\lambda T_{b_{1}}^{-}}\right]=E_{-}+\mathbb{E}\left[e^{-\lambda T_{b_{1}}^{-}} \mathbf{1}_{\left\{T_{b_{2}}^{+}<T_{b_{1}}^{-}\right\}}\right], \\
& \mathbb{E}\left[e^{-\lambda T_{b_{2}}^{+}}\right]=E_{+}+\mathbb{E}\left[e^{-\lambda T_{b_{2}}^{+}} \mathbf{1}_{\left\{T_{b_{1}}^{-}<T_{b_{2}}^{+}\right\}}\right] .
\end{aligned}
$$

We can write $\mathbb{E}\left[e^{-\lambda T_{b_{1}}^{-}} \mathbf{1}_{\left\{T_{b_{2}}^{+}<T_{b_{1}}^{-}\right\}}\right]=\mathbb{E}\left[\mathbb{E}\left[\exp \left(-\lambda\left(T_{b_{2}}^{+}+\tilde{T}_{b_{1}-Z_{T_{b_{2}}^{+}}}\right)\right) \mid \mathcal{F}_{T_{b_{2}}^{+}}\right] \mathbf{1}_{\left\{T_{b_{2}}^{+}<T_{b_{1}}^{-}\right.}\right\}$, where $\tilde{T}$ denotes a stopping time related to a Brownian motion independent of $\mathcal{F}_{T_{b_{2}}^{+}}$. Hence, we get $\mathbb{E}\left[e^{-\lambda T_{b_{1}}^{-}} \mathbf{1}_{\left\{T_{b_{2}}^{+}<T_{b_{1}}^{-}\right\}}\right]=\mathbb{E}\left[\exp \left(-\lambda T_{b_{2}}^{+}\right) \mathbf{1}_{\left\{T_{b_{2}}^{+}<T_{b_{1}}^{-}\right\}} \mathbb{E}\left[\exp \left(-\lambda\left(\tilde{T}_{b_{1}-z}^{-}\right)\right)\right]_{\left.\right|_{z=Z} T_{b_{2}}^{+}}\right]$. Since $b_{1}-Z_{T_{b_{2}}^{+}}<0$, we get

$$
\mathbb{E}\left[e^{-\lambda T_{b_{1}}^{-}} \mathbf{1}_{\left\{T_{b_{2}}^{+}<T_{b_{1}}^{-}\right\}}\right]=\frac{e^{\theta b_{1}}}{\psi(\theta \sqrt{D})} \mathbb{E}\left[\exp \left(-\lambda T_{b_{2}}^{+}\right) \mathbf{1}_{\left\{T_{b_{2}}^{+}<T_{b_{1}}^{-}\right\}} e^{-\theta Z_{T_{b_{2}}^{+}}}\right]
$$

From Chesnev et al. (1997, Sections 8.3 and 8.4) we know that $T_{b_{2}}^{+}$is an $\mathcal{F}_{g_{t}}^{+}$-stopping time whereas $Z_{T_{b_{2}}^{+}}$is independent of $\mathcal{F}_{g_{T_{b_{2}}^{+}}^{+}}$. Hence, we get $\mathbb{E}\left[e^{-\lambda T_{b_{1}}^{-}} \mathbf{1}_{\left\{T_{b_{2}}^{+}<T_{b_{1}}^{-}\right\}}\right]=$ $\frac{e^{\theta b_{1}}}{\psi(\theta \sqrt{D})} \mathbb{E}\left[e^{-\theta Z_{T_{b_{2}}^{+}}}\right]=a_{1} E_{+}$. The same kind of proof leads to $\mathbb{E}\left[e^{-\lambda T_{b_{2}}^{+}} \mathbf{1}_{\left\{T_{b_{1}}^{-}<T_{b_{2}}^{+}\right\}}\right]=a_{2} E_{-}$, which ends the proof. 


\section{Laplace transforms of single barrier Parisian option prices}

In this section, we recall the prices of single barrier Parisian options that are required to compute the double barrier Parisian option prices. In the following, $d$ denotes $\frac{b-k}{\sqrt{D}}$ and $\theta=\sqrt{2 \lambda}$. We also recall $k=\frac{1}{\sigma} \log \left(\frac{K}{x}\right)$. For more details on the computations, we refer to Labart and Lelong (2006).

\section{D.1 Standard call option}

$$
\widehat{S C}^{\star}(x, \lambda ; K ; r, \delta)=\left\{\begin{array}{cl}
\frac{K}{\theta} e^{(m-\theta) k}\left(\frac{1}{m-\theta}-\frac{1}{m+\sigma-\theta}\right) & \text { for } K \geq x, \\
\frac{2 K}{m^{2}-\theta^{2}}-\frac{2 x}{(m+\sigma)^{2}-\theta^{2}}+\frac{K e^{(m+\theta) k}}{\theta} & \text { for } K \leq x . \\
\left(\frac{1}{m+\theta}-\frac{1}{m+\sigma+\theta}\right) &
\end{array}\right.
$$

\section{D.2 Parisian down in call}

We recall that $\psi$ is defined in Definition C.1. For any $\lambda>\frac{(m+\sigma)^{2}}{2}$, it holds

$$
\begin{gathered}
\widehat{P D I C}^{\star}(x, \lambda ; K, L ; r, \delta)=\frac{\psi(-\theta \sqrt{D}) e^{2 b \theta}}{\theta \psi(\theta \sqrt{D})} K e^{(m-\theta) k}\left(\frac{1}{m-\theta}-\frac{1}{m+\sigma-\theta}\right), \\
\text { for } L<K \text { and } L \leq x . \\
\widehat{P D I C}^{\star}(x, \lambda ; K, L)=\frac{e^{(m+\theta) b}}{\psi(\theta \sqrt{D})}\left(\frac{2 K}{m^{2}-\theta^{2}}\left[\psi(-\sqrt{D} m)+\sqrt{2 \pi D} e^{\frac{D m^{2}}{2}} m \mathcal{N}(-d-\sqrt{D} m)\right]\right. \\
\left.-\frac{2 L}{(m+\sigma)^{2}-\theta^{2}}\left[\psi(-\sqrt{D}(m+\sigma))+\sqrt{2 \pi D} e^{\frac{D}{2}(m+\sigma)^{2}}(m+\sigma) \mathcal{N}(-d-\sqrt{D}(m+\sigma))\right]\right) \\
+\frac{K e^{(m+\theta) k}}{\theta \psi(\theta \sqrt{D})}\left(\frac{1}{m+\theta}-\frac{1}{m+\sigma+\theta}\right)\left[\psi(-\theta \sqrt{D})+\theta e^{\lambda D} \sqrt{2 \pi D} \mathcal{N}(d-\theta \sqrt{D})\right] \\
\quad+\frac{e^{\lambda D} \sqrt{2 \pi D}}{\psi(\theta \sqrt{D})} K e^{2 b \theta} e^{(m-\theta) k} \mathcal{N}(-d-\theta \sqrt{D})\left(\frac{1}{m+\sigma-\theta}-\frac{1}{m-\theta}\right) \\
\text { for } K \leq L \leq x .
\end{gathered}
$$




\section{D.3 Parisian up in call}

For any $\lambda>\frac{(m+\sigma)^{2}}{2}$, it holds

$$
\begin{gathered}
\widehat{P U I C}^{\star}(x, \lambda ; K, L ; r, \delta)=e^{(m-\theta) b} \frac{\sqrt{2 \pi D}}{\psi(\theta \sqrt{D})}\left[\frac{2 K}{m^{2}-\theta^{2}} e^{\frac{D m^{2}}{2}} m \mathcal{N}(d+\sqrt{D} m)\right. \\
\left.\quad-\frac{2 L}{(m+\sigma)^{2}-\theta^{2}} e^{\frac{D(m+\sigma)^{2}}{2}}(m+\sigma) \mathcal{N}(d+\sqrt{D}(m+\sigma))\right] \\
+\frac{e^{-2 b \theta}}{\psi(\theta \sqrt{D})} K e^{(m+\theta) k} e^{\lambda D} \sqrt{2 \pi D} \mathcal{N}(d-\theta \sqrt{D})\left(\frac{1}{m+\sigma+\theta}-\frac{1}{m+\theta}\right) \\
+\frac{e^{(m-\theta) k}}{\theta \psi(\theta \sqrt{D})} K\left(\frac{1}{m-\theta}-\frac{1}{m+\sigma-\theta}\right)\left(\psi(-\theta \sqrt{D})+\theta \sqrt{2 \pi D} e^{\lambda D} \mathcal{N}(d-\theta \sqrt{D})\right) \\
\quad \text { for } x \leq L \leq K . \\
\widehat{P U I C}^{\star}(x, \lambda ; K, L ; r, \delta)=\frac{e^{(m-\theta) b}}{\psi(\theta \sqrt{D})}\left[\frac{2 K}{m^{2}-\theta^{2}} \psi(\sqrt{D} m)-\frac{2 L}{(m+\sigma)^{2}-\theta^{2}} \psi(\sqrt{D}(m+\sigma))\right] \\
+\frac{e^{-2 b \theta} \psi(-\theta \sqrt{D})}{\theta \psi(\theta \sqrt{D})} K \mathrm{e}^{(m+\theta) k}\left(\frac{1}{m+\theta}-\frac{1}{m+\theta+\sigma}\right)
\end{gathered}
$$

\section{Acknowledgments}

We wish to thank B. Lapeyre for thoroughly reading the paper. We also would like to address our deepest gratitude to A. Alfonsi for the numerous comments he made on a previous version of this work and the fruitful discussions we had about complex analysis.

\section{References}

J. Abate and P. Valko. Multi precision laplace transform inversion. International Journal of Numerical Methods in Engineering, 60(5-7):979-993, 2004.

J. Abate and W. Whitt. The Fourier-series method for inverting transforms of probability distributions. Queueing Systems Theory Appl., 10(1-2):5-87, 1992. ISSN 0257-0130.

J. Abate, L. Choudhury, and G. Whitt. An introduction to numerical transform inversion and its application to probability models. Computing Probability, pages 257 - 323, 1999.

J. Anderluh and J. Van der Weide. Parisian options - the implied barrier concept. International Conference on Computational Science 2004, pages 851-858, 2004.

L. Andersen and R. Brotherton-Ratcliffe. Exact exotics. Risk, 9(10):85-89, 1996.

M. Avellaneda and L. Wu. Pricing Parisian-style options with a lattice method. International Journal of Theoretical and Applied Finance, 2(1):1-16, 1999. 
P. Baldi, L. Caramellino, and M. G. Iovino. Pricing complex barrier options with general features using sharp large deviation estimates. In Monte Carlo and quasi-Monte Carlo methods 1998 (Claremont, CA), pages 149-162. Springer, Berlin, 2000.

C. Bernard, O. LeCourtois, and F. Quittard-Pinon. A new procedure for pricing Parisian options. The Journal of Derivatives, 12(4):45-53, 2005.

M. Chesney, M. Jeanblanc-Picqué, and M. Yor. Brownian excursions and Parisian barrier options. Adv. in Appl. Probab., 29(1):165-184, 1997. ISSN 0001-8678.

M. Costabile. A combinatorial approach for pricing Parisian options. Decis. Econ. Finance, 25(2):111-125, 2002. ISSN 1593-8883.

P. Forsyth and K. Vetzal. Discrete parisian and delayed barrier options: A general numerical approach. Adv. Futures Options Research, 10:1-16, 1999.

R. Haber, P. Schonbucher, and P.Wilmott. An American in Paris. OFRC Working Papers Series 1999mf14, Oxford Financial Research Centre, 1999. available at http://ideas.repec.org/p/sbs/wpsefe/1999mf14.html

P. Hartley. Pricing Parisian options by Laplace inversion. Decisions in Economics $\mathcal{E}$ Finance, 2002.

C. Labart and J. Lelong. Pricing Parisian options. Technical report, ENPC, http://cermics.enpc.fr/reports/CERMICS-2005/CERMICS-2005-294.pdf December 2005.

C. Labart and J. Lelong. Pricing Parisian options using Laplace transforms. Technical report, ENPC, http://cermics.enpc.fr/reports/CERMICS-2006/CERMICS-2006-328.pdf November 2006.

J. Pitman and M. Yor. The two-parameter Poisson-Dirichlet distribution derived from a stable subordinator. Ann. Probab., 25(2):855-900, 1997. ISSN 0091-1798.

M. Schröder. Brownian excursions and Parisian barrier options: a note. J. Appl. Probab., 40 (4):855-864, 2003. ISSN 0021-9002.

D. V. Widder. The Laplace Transform. Princeton Mathematical Series, v. 6. Princeton University Press, Princeton, N. J., 1941.

P. Wilmott. Derivatives. University Edition, 1998. 